\title{
Unusual Cause of Sphenoidal Sinus Mass: Extramedullary Plasmacytoma
}

\author{
Gokhan Kuyumcu, Robert J. Dawe, Miral Jhaveri
}

doi:10.1017/cjn.2016.319

Can J Neurol Sci. 2017; 44: 324-325

Plasmacytomas are classified into three groups: 1) plasmacytomas accompanying evident multiple myeloma with generalized bone involvement and characteristic radiographic findings; 2) solitary plasmacytoma of the bone with no evidence of generalized disease; and 3) primary plasmacytoma of the soft tissues. ${ }^{1}$ Solitary plasmacytomas are rare, accounting for $4 \%$ of all plasma cell diseases. $^{2}$ Extramedullary plasmacytomas are even rarer; they represent less than $1 \%$ of all head and neck malignancies and less than $0.4 \%$ of upper respiratory malignancies. Primary extramedullary plasmacytomas most commonly occur in the head and neck region, with the upper respiratory tract and oral cavity affected primarily. Salivary glands, skin, cervical lymph nodes, orbit, thyroid gland, and larynx can also be affected. ${ }^{3}$ Submucosal tissue in the upper aerodigestive tract has resident plasma cells. Primary plasma cell tumors
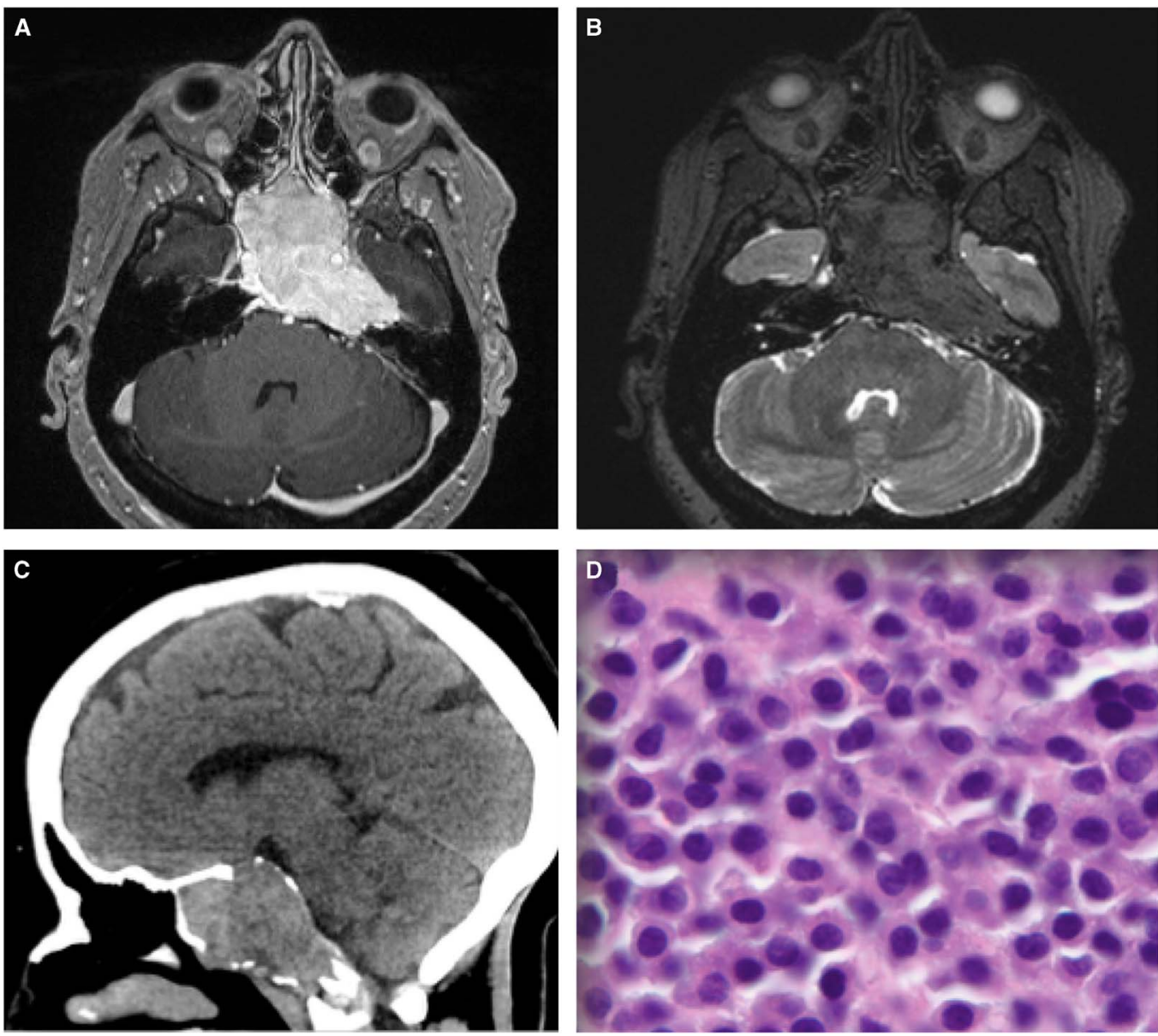

Figure 1: (A) MRI of plasmacytomas show avid enhancement in addition to (B) hypo-isointense areas on T2 weighted images due to hypercellularity. (C) CT shows bulky mass causing bone erosion. (D) Hematoxylin and Eosin-stained sections of core biopsy revealed only monotonous sheets of plasma cells. 
of this region is believed to be caused by proliferation of the local plasma cell pool, leading to plasmacytomas. ${ }^{4}$ Plasmacytomas are more common in males, with a male-to-female ratio of 4 to 1 . They most commonly present as a solitary lesion after the fifth or sixth decade of life. Extramedullary plasmacytomas presenting with sphenoidal sinus mass are extremely rare; the literature consists of several case reports and a case series. ${ }^{4-6}$

A 67-year-old female patient presented with a chief complaint of left nasal obstruction for two months, and blood tinged mucus and epistaxis for one week. Physical examination showed left hypoglossal nerve paralysis. Prescribed antibiotics and intranasal steroid inhalers did not improve the symptoms. Nasal endoscopy revealed a soft, polypoid mass occupying the left nasal cavity. CT showed a large mass filling the sphenoid sinus, eroding the sella floor, and involving the left hypoglossal canal. On MRI the mass had intrinsic T2 iso-hypointensity and showed avid enhancement. Radiological differentials included invasive pituitary adenoma, lymphoma, meningioma, and chordoma. The CT scan of the lungs and abdomen was negative. Hematoxylin and Eosin-stained sections of core biopsy revealed only monotonous sheets of plasma cells (Figure 1D). Serum and urine electrophoresis tests failed to demonstrate any myeloma component or Bence-Jones protein. Bone marrow biopsy results were negative.

Immunohistochemical staining showed diffuse kappa light chain staining of cytoplasma and CD 138 staining of membrane. The final pathologic diagnosis was an extramedullary plasmacytoma. Radiological differential considerations included invasive pituitary adenoma, lymphoma, meningioma, and possibly metastasis. Bulky mass causing bone erosions is the most common finding of extramedullary plasmacytomas and skull base plasmacytomas on CT (Figure 1C). MR findings show typically hypo-isointense areas on T2 weighted images due to hypercellularity and avid enhancement (Figure 1A, B). Spheno-occipital chordoma was one of the top differentials in our case, due to clivus involvement, bone destruction, and bulky mass. We did not. however, see T2 hyperintensity and amorphous intratumoral calcifications, which are the most helpful clues for diagnosis of chordoma. Pituitary adenomas can show T2 isointensity, but they typically show delayed enhancement, and avid enhancement is extremely uncommon. Differential diagnoses for an avidly enhancing, T2 iso-hypointense skull base mass with bone erosion should include solitary plasmacytoma. Though rare, they most commonly present as a skull base mass. ${ }^{7}$

\section{Statement of Authorship}

Gokhan Kuyumcu: Drafting the article, approval of the final version.

Miral Jhaveri: Conception and design, approval of the final version.

Robert Dawe: Critical revision for important intellectual content, approval of the final version.

\section{DisCLOSURES}

Gokhan Kuyumcu, Robert Dawe, and Miral Jhaveri do not have anything to disclose.

\section{REFERENCES}

1. Shreif JA, Goumas PD, Mastronikolis N, Naxakis SS. Extramedullary plasmacytoma of the nasal cavity. OtolaryngologyHead and Neck Surgery. 2001;124(1):119-20.

2. Chang YL, Chen PY, Hung SH. Extramedullary plasmacytoma of the nasopharynx: A case report and review of the literature. Oncology letters. 2014;7(2):458-60.

3. Corvo MA, Granato L, Ikeda F, de Prospero JD. Extramedullary nasal plasmacytoma: Literature review and a rare case report. International Archives of Otorhinolaryngology. 2013;17(2):213-7.

4. Lomeo PE, McDonald JE, Finneman J. Extramedullary plasmacytoma of the nasal sinus cavities. American Journal of Otolaryngology. 2007;28(1):50-1.

5. Marom T, Goldfarb A, Vaknine H, Kravtsov V, Roth Y. Sinonasal extramedullary plasmacytoma. Otolaryngology-Head and Neck Surgery. 2009;141(4):533-4.

6. Özdemir S, Tarkan Ö, Tuncer Ü, Sürmelioğlu Ö, Doğrusöz M, Ergin M. A case of extramedullary plasmacytoma in the sphenoid sinus with unilateral loss of vision. Journal of Cranio-Maxillofacial Surgery. 2013;41(2):140-3.

7. Agarwal A. Neuroimaging of plasmacytoma. A pictorial review. The neuroradiology. Journal. 2014;27(4):431-7. 